\title{
Voluntary attention and the spacing effect
}

\author{
DOUGLAS L. HINTZMAN, JEFFERY J. SUMMERS, NORMAN T. EKI, and MARLENE D. MOORE \\ University of Oregon, Eugene, Oregon 97403
}

\begin{abstract}
Three experiments were done to test the hypothesis that the spacing effect results from a voluntary decision by the subject to pay little attention to the second presentation $\left(P_{2}\right)$ of an item when it occurs shortly after the first $\left(P_{1}\right)$. In all three experiments, the spacing of repetitions was varied. In Experiment $I$, allocation of attention was manipulated by pairing $P_{2}$ of some pictures with a signal that indicated high payoff for later retention. In Experiment II, attention was controlled more directly by requiring the subject, in one condition, to recite words aloud. In both experiments, the dependent variable was judged frequency. In neither experiment did the effect of the attention manipulation interact with that of the spacing of repetitions. In Experiment III, the number of eye fixations given a picture was taken to be a measure of attention. The number of fixations dropped from $P_{1}$ to $P_{2}$ to $P_{3}$, but was unaffected by the spacing of repetitions. The experiments provide no support for a voluntary attention explanation of the spacing effect.
\end{abstract}

If two presentations of a stimulus item $\left(\mathrm{P}_{1}\right.$ and $\left.\mathrm{P}_{2}\right)$ occur in close succession, later retention of the item is poorer than it is if the two presentations are spaced further apart. Several different hypotheses have been proposed to explain this effect (for a review, see Hintzman, 1974). One view holds that when $P_{2}$ occurs shortly after $P_{1}$, the subject gives it less attention than when the repetition is delayed. This explanation makes two basic assumptions: first, that the spacing effect is due to deficient processing of $P_{2}$ when the $P_{1}-P_{2}$ lag is short, and second, that the degree of processing of $P_{2}$ is deficient because of a voluntary decision by the subject regarding the allocation of attention.

This paper reports three experiments designed to test predictions of the voluntary attention explanation of the spacing effect. In Experiment I, the stimuli were scenic pictures and the dependent variable was judged frequency. An attempt was made to control indirectly the attention subjects gave the stimuli by presenting, along with selected pictures, a tone which signaled that there would be a high payoff for remembering the picture on a later test. In accordance with the voluntary attention hypothesis, it was predicted that the magnitude of the spacing effect would be less when $\mathrm{P}_{2}$ was accompanied by a tone than when it was not. A manipulation that increases the degree of attention given a stimulus should have its greatest effect on performance in conditions in which the attenuation of attention is ordinarily greatest. According to the hypothesis, the attenuation of attention is greatest at short $P_{1}-P_{2}$ spacings, so that is where the tone should have its greatest facilitative effect.

In Experiment II, more direct control over the subjects' processing activities was sought. The stimuli

These investigations were supported by a research grant from the Office of Education, United States Department of Health, Education, and Welfare, and by Grant GB-40360 from the National Science Foundation. Requests for reprints should be addressed to Douglas L. Hintzman, Psychology Department. University of Oregon, Eugene, Oregon 97403. were words and, in one condition, the subjects were instructed to recite a word aloud three times on each presentation. In the other condition, the subjects were free to study the words silently, in any way they wished. The dependent variable was judged frequency, as in Experiment I. The prediction was that the magnitude of the spacing effect would be less in the condition in which allocation of attention was restricted by experimental instructions than in the condition in which it was not.

In Experiment III, an attempt was made to measure attention directly during study of the list. Stimuli were scenic pictures, and the number of eye fixations made to each stimulus was recorded. The prediction was that the number of fixations made to a repetition of a picture, an overt indicator of interest (Loftus, 1972), would increase with the length of the prior spacing interval.

\section{EXPERIMENT I}

\section{Method \\ Materials and design. The experimental items were color slides of 170 different vacation scenes depicting landscapes, buildings, people, automobiles, etc. They were randomly assigned to 12 different conditions. In one condition, there were 50 pictures which had a frequency of zero $(F=0)$ during the presentation series, and served as distractor items on the frequency-judgment test. Another 40 pictures occurred with a frequency of one $(F=1)$ during the presentation series. Twenty were accompanied at presentation onset by a tone and 20 were not. The remaining 80 slides occurred with a frequency of two $(F=2), 10$ in each of 8 conditions. The 8 conditions represented the orthogonal combination of two incentive values (either a tone or no tone at the onset of $\left.P_{2}\right)$ and four levels of $P_{1}-P_{2}$ spacing $(S=0,1,5$, and 15 intervening items). \\ The presentation series was divided into 10 blocks. In each block, each of the eight $F=2$ conditions was represented by 1 picture, and the two $F=2$ conditions were represented by 2 pictures each. One additional picture was presented three times in each block at spacing intervals that varied, and was never accompanied by the tone. The within-block order of conditions was random, subject to the limitations imposed by spacing requirements of the conditions. In addition, the first 10 and last}




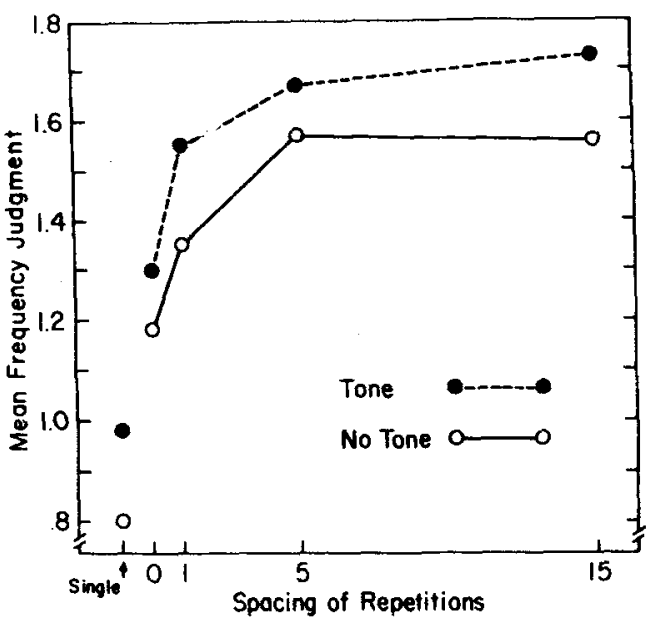

Figure 1. Effects of the incentive tone on judged frequency of pictures. Isolated points: single-presentation pictures with and without the tone. Curves: the spacing effect with and without a tone at $\mathbf{P}_{2}$.

10 slides in the entire series were filler items drawn from the same stimulus pool. Some of the filler slides were repetitions. Altogether there were 250 slides, including repetitions and fillers, in the entire presentation series.

The frequency-judgment test series, which was composed of 170 experimental slides, was divided into 5 blocks of 34 slides each. Each $F=2$ condition was represented by 2 pictures and each $F=1$ condition by 4 pictures in each block. The remaining 10 slides were $F=0$ items. The within-block order of the slides was random, and each block of the presentation series was represented approximately equally in each block of the test series. The test sheet on which subjects marked their frequency judgments consisted of 170 consecutively numbered blank lines.

Subjects and procedure. The subjects were 45 paid volunteers obtained through an advertisement in the University of Oregon campus newspaper. They were tested in four sessions of 9 to 12 subjects each. Across sessions, slides were partially rotated through conditions within frequency levels and within blocks, in such a way that overall, each picture occurred both with and without a tone, and $F=2$ pictures occurred equally often at each level of $P_{1}-P_{2}$ spacing.

At the outset of each session, subjects were informed that a series of 250 slides would be shown for 3 sec each, that some of the slides would be repeated, and that they were to study the slides for a later memory test, the nature of which was not specified. They were also told that some pictures would be accompanied by a tone, and that the tone indicated that the picture being shown was of particular importance and that they should make a special attempt to memorize it. The subjects were further informed that later in the experiment they would be paid according to the number of pictures remembered, with pictures that were accompanied by a tone worth four times as much as those that were not.

The slide series was then presented by a Kodak Carousel projector which was controlled by a stereophonic tape recorder and Kodak sound synchronizer. Pulses to control the projector had been prerecorded on one channel of the tape by computer. On the other channel wete recorded brief audio signals, timed to be simultaneous with onset of the appropriate slides. Each slide was presented for $2.2 \mathrm{sec}$ with a $0.8-\mathrm{sec}$ interstimulus interval.

Following presentation, the test sheets were distributed. The subjects were told that a test series would be shown, one picture at a time, and that they were to judge how many times each picture had occurred in the previous list, guessing if uncertain. If they did not remember a picture as having occurred, thay were to give a judgment of zero. Instructions stated that they would be paid $\$ .04$ for a correct frequency judgment given to a picture that had been accompanied by a tone during presentation, and $\$ .01$ for each correct frequency judgment given to other pictures. The test series was then presented at a $5-\sec$ rate. To ensure that subjects wrote their judgments on the correct line of the test sheet, the experimenter called out the number of each slide as it was presented.

\section{Results}

Mean judged frequency for condition $F=0$ was 0.22 . The judgments for the other conditions are presented in Figure 1. The two data points in the lower left of the figure demonstrate the effect of the incentive manipulation on memory for pictures that occurred one time. Mean judged frequency was higher for pictures that had been accompanied by the tone than for those that had not $[\mathrm{F}(1 / 44)=32.1, \mathrm{p}<.001, \mathrm{MSE}=.084]$.

The effect of spacing on judged frequency was tested using a planned comparisons analysis of variance. The comparison coefficients used for spacings of $0,1,5$, and 15 intervening items, respectively, were: $-3,-1,+2$, and +2 . These values were chosen to be consistent with the observation that the spacing effect reaches asymptote at about a $15-\sec P_{1}-P_{2}$ interval (Hintzman, 1974). The spacing comparison was reliable for both the tone and no tone curves individually $[F(1 / 44)=85.0$ (MSE $=.053)$ and $74.7 \quad(\mathrm{MSE}=.063)$ respectively, $p<.001]$. Most important for the voluntary attention hypothesis is the fact that the two curves have practically the same form. The interaction of spacing with the incentive manipulation was not significant, $\mathrm{F}<1$. Thus the prediction that the tone should have its greatest effect at short spacings, where the attenuation of attention is supposedly the greatest, was not supported.

Although the incentive manipulation used here apparently did have the desired effect on attention, the form of control used was weak. The processing strategy was still determined by the subject, and it could be that the influence of the $P_{1}-P_{2}$ interval on the subject's decision to attend is more powerful than the influence of the instruction regarding monetary incentive. In Experiment II, more direct control was exercised. Words were used as stimuli, and subjects were required to say them aloud.

\section{EXPERIMENT II}

\section{Method}

Materials and design. The experimental materials were 120 common three-letter English nouns. They were typed using a bulletin typewriter and photographed, and the negatives were mounted in slide frames. Thirty-six words were assigned at random to each of three frequencies of occurrence $(F=1,2$, and $3)$, and 12 were assigned to the distractor condition ( $F=0)$. Within the $F=2$ and $F=3$ conditions, 12 words were assigned at random to each of three spacings $(S=0,1$, and 5 intervening items). 
The presentation sequence consisted of 240 slides, arranged in three Kodak Carousel slide trays. The first five and last three slides of each tray were filler items. The 72 experimental slides for each tray were arranged, with appropriate spacings, in three overlapping blocks. All conditions except $F=0$ (which occurred only on the test) were evenly distributed over the three blocks. Two additional sequences were constructed by rotating words in the $F=2$ and $F=3$ conditions across the three levels of spacing. Thus, there were three different rotations of the stimulus sequence, presented to different subjects.

The primary manipulation was study method. Of the three slide trays, each subject studied the words in one silently, and the words in the other two aloud. In the latter method, subjects were instructed to recite each word aloud three times, at the rate of about once per second. The designation of which slide tray was to be studied silently (Tray 1,2 , or 3 ) varied orthogonally with the rotation of items among conditions. Thus, each rotation of each tray occurred in both the Silent and Aloud conditions.

A single test form was used for all subjects. It consisted of a mimeographed list of all the $F=2$ and $F=3$ words, and 12 each of the $F=1$ and $F=0$ words, making a total of 96 test items. To the right of each word was a blank line for the subject's frequency judgment. The words were arranged in six blocks, with each of the eight experimental conditions occurring equally often in each block.

Subjects and procedure. There were 42 subjects altogether. Data from 6 of them were dropped due to failure to follow instructions and to equipment malfunctions. Of the remaining 36,18 were volunteers from an undergraduate psychology class serving for credit, and 18 were paid volunteers from University of Oregon dormitories. They were tested individually. The designation of which of the three trays was to be studied silently was rotated after each subject (this variable defined three groups of 12 subjects each), and items were rotated among spacings after every sixth subject had been tested.

At the outset of the experiment, the subject was told that a series of words would be projected on the wall, that some of the words would be repeated, and that he was simply to study each word for as long as it was presented and try to remember it for a later test, the nature of which was not specified. The three trays of slides were presented separately, with short breaks between trays. Immediately before being shown a tray of slides, the subject was told which study method (silent or aloud) was to be used with that tray. The only exception was that when Tray 1 was a silent tray, no special instruction was given, as subjects always naturally studied it silently. The tray of 80 slides was then presented using a Carousel projector paced by a timer at a 3-sec rate.

After all three slide trays had been presented, the subject was given a test form and was instructed to write in the blank next to each word the number of times he thought the word had occurred in the study series.

\section{Results}

The mean judged frequency for $F=0$, which did not occur in the list, was 0.43 . Mean judgments of frequency for the other conditions are shown as a function of spacing and study method in Figure 2. The data for presentation frequencies $F=1,2$, and 3 are presented separately in different panels. The figure shows that judged frequencies were uniformly higher to words that had been recited aloud than to words that were studied silently, and that judged frequency increased with spacing under both study methods. There is no evidence to support the prediction that the magnitude of the spacing effect would be less in the aloud than in the silent condition.

An analysis of variance using planned comparisons supported the above observations. The linear trend of frequency judgments on frequency was significant $[\mathrm{F}(1 / 33)=296, \mathrm{p}<.001, \mathrm{MSE}=.153]$, as was the difference between silent and aloud study methods $[\mathrm{F}(1 / 33)=14.4, \mathrm{p}<.001, \mathrm{MSE}=.503]$. The latter difference was not reliably affected by whether the tray that was studied silently was first, second, or third, and this is shown by the lack of a significant Silent vs Aloud by Groups interaction $[F(2 / 33)=3.28, \quad p>.05$, MSE $=.503]$. The spacing effect was tested, using comparison coefficients $-3,+1$, and +2 for spacings of $\mathrm{S}=0,1$, and 5 intervening items, respectively. The effect was significant under both the silent and aloud study methods $[F(1 / 33)=17.5 \quad(\mathrm{MSE}=.235)$ and 23.1 (MSE = .139), respectively, both $\mathrm{p}<.001]$. The interaction of spacing and study method was not significant, $\mathrm{F}<1$.

The outcome of Experiment II was thus consistent with that of Experiment $I$ in failing to confirm the prediction of an interactive relationship between the effect of a manipulation of attention and the effect of spacing. In Experiment III, the effect of spacing on attention was measured, using eye fixations as an index of attention.

\section{EXPERIMENT III}

\section{Method}

Materials. The experimental stimuli were color vacation slides from the same population as those used in Experiment I. Fourteen different pictures were assigned to each of three different spacing conditions, $\mathrm{S}=0,1$, and 5 intervening items (or alternatively, $0.8,5.8$, and $25.8 \mathrm{sec}$ ). There were three presentations of each picture, with the $P_{1}-P_{2}$ and $P_{2}-P_{3}$ spacings for a given picture the same. The slides were arranged in two 80 -slide trays, in a pattern in which pictures from the three spacing conditions were distributed about equally over serial positions. The pattern for Tray 2 was essentially the mirror image of that of Tray 1 . In either tray there were seven pictures from each of the three spacing conditions (three slides of each

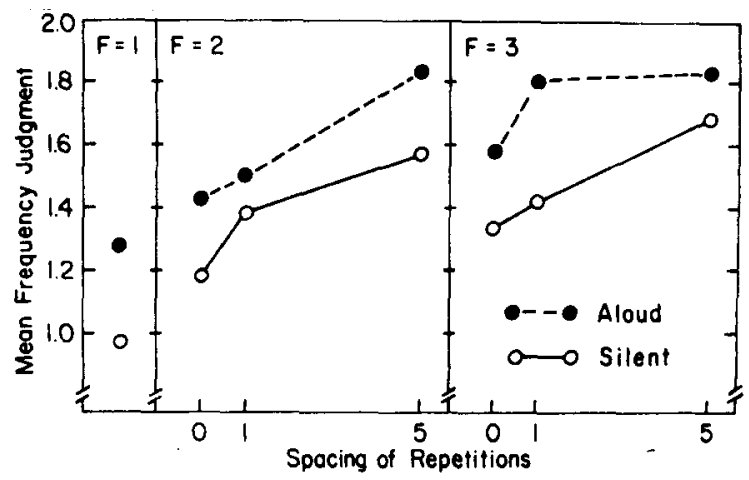

Figure 2. Effects of study method on judged frequency of words. Different presentation frequencies appear in different panels. 
Table 1

Mean Numbers of Eye Fixations Per 5 Sec as a Function of Frequency and Spacing: Experiment III

\begin{tabular}{cccc}
\hline & \multicolumn{3}{c}{ Spacing (Seconds) } \\
$\begin{array}{c}\text { Presen- } \\
\text { tation }\end{array}$ & .8 & 5.8 & 25.8 \\
\hline $\mathrm{P}_{1}$ & 6.57 & 6.41 & 6.68 \\
$\mathrm{P}_{2}$ & 5.92 & 5.81 & 5.77 \\
$\mathrm{P}_{3}$ & 5.58 & 5.52 & 5.54 \\
\hline
\end{tabular}

picture), plus 17 filler slides. The first three slides of either tray were fillers.

Subjects and procedure. The data from six subjects, all volunteers from two undergraduate psychology classes at the University of Oregon, were used. The data of one other subject were discarded due to technical difficulties. The subjects were tested individually. Between sessions, pictures were rotated through the three spacing conditions, with two subjects tested on each of three different rotations.

The subjects were seated, with their heads held stationary by a chin rest, 21 in from a back projection screen. The pictures were projected by a Kodak Carousel projector, and on the screen they measured $12 \times 18$ in. A Sony videotape camera equipped with a $75-\mathrm{mm}$ zoom lens and $2 x$ extender was stationed 27 in from the subject's face, and was focused on the left eye.

The subjects were told that they were participating in a memory experiment, and that a series of pictures would be presented on the screen at 5 -sec intervals. They were instructed to give full attention to the pictures, some of which would be repeated several times, and to try to remember them for a later test. The purpose of the experiment, the subjects were told, was to determine the relationship between pupil size and memory-hence the camera.

The first slide tray was then presented at a $5-\mathrm{sec}$ rate. Actual exposure duration was $4.2 \mathrm{sec}$, and the interstimulus interval was $0.8 \mathrm{sec}$. A continuous picture of the subject's left eye was recorded on the video channel of the tape. Simultaneously, the sound of the slide-change apparatus was recorded on the audio channel, to delineate the onset and offset of each picture for the analysis of eye movements. Following presentation of Tray 1 , a brief rest was given. Tray 2 was then presented in the same manner. No memory test was given.

\section{Results}

The video tapes were played back at normal speed, and the number of eye fixations made to each picture was counted independently by two observers. These counts were blind with respect to conditions and to the counts made by the other observer. The correlations between the two observers' tallys ranged from 669 to .822 for the six subjects who were retained (that for the subject whose data were dropped was .553). The mean was .730 and the standard deviation was .066 . While these correlations are not particularly impressive, they are sufficiently high that the means of the two observers' counts should correlate over .90 with the criterion.

The mean number of eye fixations made during each $5-\mathrm{sec}$ presentation interval is shown as a function of spacing and presentation number (frequency) in Table 1. Number of fixations was affected by repetition in the way that would be expected if familiar stimuli attracted less attention than novel ones. Overall mean numbers of fixations were: $P_{1}, 6.55 ; P_{2}, 5.83 ; P_{3}, 5.55$. The spacing of repetitions, by contrast, had no noticeable effect on fixations. The cumulative number of fixations over three presentations was 18.07 when the spacing was $0.8 \mathrm{sec}$, 17.74 when it was $5.8 \mathrm{sec}$, and 17.99 when it was $25.8 \mathrm{sec}$. These values are nearly identical, and in any case the differences are in the wrong direction to be consistent with the voluntary attention explanation of the spacing effect.

In a 3 by 3 analysis of variance with repeated measures, only the effect of frequency was reliable $[F(2 / 10)=18.03, p<.001, \mathrm{MSE}=.268]$. Neither the effect of spacing nor the Frequency by Spacing interaction reached significance, $\mathrm{F}<1$.

\section{DISCUSSION}

The results of these three experiments provide no support for the voluntary attention account of the spacing effect. In Experiment I, where attention given $\mathrm{P}_{2}$ was controlled indirectly through an incentive manipulation, the effect of the incentive did not interact with that of spacing. Likewise, in Experiment II, where attention was controlled more directly by requiring subjects to recite the words aloud, the attention manipulation and the spacing effect did not interact. In both experiments, the interaction predicted by the voluntary attention hypothesis was one in which the spacing effect is less evident or absent when subjects are induced to give full attention to a repeated item. And in Experiment III, where the number of eye fixations was taken to be an overt index of attention to pictures, there was no evidence that attention to repetitions was affected by spacing.

How do the present conclusions relate to the findings of previous investigations? Despite the popularity of the voluntary attention hypothesis, there is little empirical support for it in the literature, and the support that exists appears weak.

In a study reported by Elmes, Sanders, and Dovel (1973), attention was manipulated by "isolating" some words in a free recall list by presenting them in a distinctive way. Isolated words were recalled better than nonisolated words (the Von Restorff effect), as expected. But recall of isolated words was affected by spacing at least as much as was that of nonisolated words. Thus, despite the contrary conclusions of Elmes et al., isolation and spacing did not interact in the way that would support the voluntary attention explanation of the spacing effect. The result was entirely consistent with those of the present Experiments I and II.

A spacing experiment by D'Agostino and DeRemer (1973, Exp. I) attempted to control attention subjects gave to sentences by requiring them to form a visual image of the subject-object interaction depicted by each sentence and to describe the image to the experimenter. The spacing of repetitions of the sentences was varied. 
The results of the study, however, are difficult to interpret. When subjects were given a cued recall test in which the subject phrase of a sentence was the cue for recall of the object phrase, no spacing effect was evident. But when other subjects were asked to free recall the object phrases of the sentences, a spacing effect was obtained. The distinct possibility of a ceiling effect in the results of the cued recall test, together with the contrary result obtained using free recall, render the support of the voluntary attention hypothesis provided by the cued recall result equivocal. This is especially true since in the present Experiment II, where a similar manipulation was used to force subjects to attend, the spacing effect was not influenced by the attention manipulation.

An experiment similar in intent to the present Experiment III was done by Shaughnessy, Zimmerman, and Underwood (1972). They allowed subjects to control the pace of presentation of a word list in which frequency and spacing were varied, and the amount of time subjects chose to spend studying each item was recorded. The amount of study time decreased with repetition, and the decrease was greater when repetitions were massed than when they were spaced. This outcome suggests that spacing does affect attention. However, eye fixations, in the present investigation, showed only an effect of frequency, and no effect of spacing.

There seems little reason to doubt that eye fixations are an index of attention, as we have assumed. Loftus (1972) showed that an incentive manipulation (similar to that of the present Experiment 1) affects the number of times a picture is fixated. And the decrease in fixations with repetition found here also supports the interpretation that fixations reflect attention. Yet an increase in spacing over the range that has the greatest effect on retention had no corresponding effect on eye fixations.

The reason for the apparent contradiction between the Shaughnessy et al. (1972) study and the present one is not clear. However, it should be noted that Shaughnessy et al. used words as stimuli, and the spacing intervals in their "distributed" condition were longer than those used here. Thus there may have been some forgetting of $P_{1}$ by the time $P_{2}$ occurred, and this might account for the effect of $P_{1}-P_{2}$ interval on study times.
In any case, they concluded that the massed vs spaced difference in study times was not great enough to account for the difference in performance on the retention test. This fact, together with the present results, suggests that the effect of spacing on voluntary study time found by Shaughnessy et al. (1972) has nothing to do with the effect of spacing on later retention.

As has been noted elsewhere (Hintzman, 1974), the spacing effect is remarkably robust over task variations. This is evident in the range of stimulus materials (words, nonsense syllables, sentences, pictures), dependent variables (recall and recognition accuracy, recognition latency, judged frequency), and tasks (paired associates, free recall, recognition memory, the distractor task) in which the effect is found. (To the list of tasks can now be added verbal discrimination learning; Ciccone, 1973). It seems unlikely that a phenomenon that appears in so many different experimental situations can be due to a voluntary decision by the subject to process information in a particular way. The ubiquity of the phenomenon, together with the lack of a relationship between manipulated study strategies and the magnitude of the spacing effect, can be considered evidence not only against the voluntary attention hypothesis, but against other control-process explanations of the spacing effect, as well.

\section{REFERENCES}

Ciccone, D. S. Massed and distributed item repetition in verbal discrimination learning. Journal of Experimental Psychology 1973, 101, 396-397.

D'Agostino, P. R., \& DeRemer, P. Repetition effects as a function of rehearsal and encoding variability. Journal of Verbal Learning and Verbal Behavior, 1973, 12, 108-113.

Elmes, D. G., Sanders, L. W., \& Dovel, J. C. Isolation of massedand distributed-practice items. Memory \& Cognition, 1973, 1, $77-79$

Hintzman, D. L. Theoretical implications of the spacing effect. In R. L. Solso (Ed.). Theories in cosnitive poychology: The Loyola symposium. Potomec, Md: Lawrence Erdbaum Associates, 1974, Pp. 7799.

Loftus, G. R. Eye fixations and recognition memory for pictures. Cognitive Psy chology, 1972, 3, 525-551.

Shaughnessy, J. J., Zimmerman, J., \& Underwood, B. J. Further evidence on the MP-DP effect in free recall learning. Journal of Verbal Learning and Verbal Behavior, 1972, 11, 1-12.

(Received November 1,1974 . (Revision received December $16,1974$. ) 